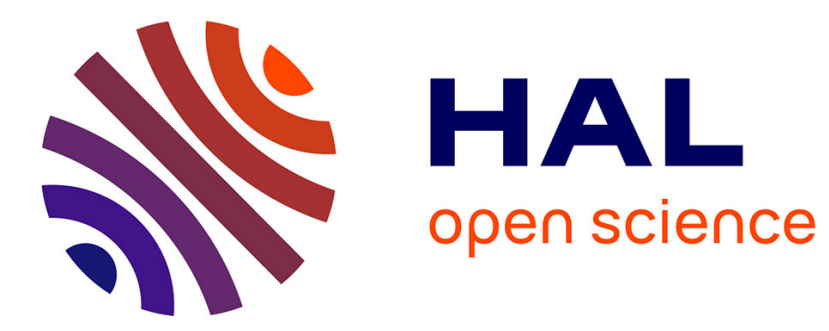

\title{
Reducing sensitivity analysis time-cost of compound model
}

\author{
Benoît Delinchant, Frédéric Wurtz, Eric Atienza
}

\section{To cite this version:}

Benoît Delinchant, Frédéric Wurtz, Eric Atienza. Reducing sensitivity analysis time-cost of compound model. IEEE Transactions on Magnetics, Mar 2004, France. pp.1216 - 1219. hal-00333824

\section{HAL Id: hal-00333824 \\ https://hal.science/hal-00333824}

Submitted on 24 Oct 2008

HAL is a multi-disciplinary open access archive for the deposit and dissemination of scientific research documents, whether they are published or not. The documents may come from teaching and research institutions in France or abroad, or from public or private research centers.
L'archive ouverte pluridisciplinaire HAL, est destinée au dépôt et à la diffusion de documents scientifiques de niveau recherche, publiés ou non, émanant des établissements d'enseignement et de recherche français ou étrangers, des laboratoires publics ou privés. 


\title{
Reducing Sensitivity Analysis Time-Cost of Compound Model
}

\author{
Benoit Delinchant, Frédéric Wurtz, and Eric Atienza
}

\begin{abstract}
This paper deals with the sensitivity analysis of compound models in the case of gradient based optimization. Multidisciplinary optimization (MDO) may use time-consuming analysis such as the finite-element method (FEM) resolution, their sensitivity analysis must then be managed efficiently in order to limit their evaluations. A composition model implementation based on differential propagation mechanism has been used. Different solutions of sensitivity analysis based on forward finite difference are proposed at the level of each inner model. These solutions have been implemented for the design of a transformer, using mixed modeling (FEM + analytic). It has led to a reduction by a factor of two then three of an optimization iteration time cost.
\end{abstract}

Index Terms-Finite difference, mixed model, optimization, sensitivity computation, transformer design.

\section{INTRODUCTION}

$\mathbf{O}$ PTIMIZATION of numerical models is a great challenge for the computer-aided design, but has still to be improved. The cost-fidelity tradeoff has to be managed to face the increasing design variable number and disciplinary coupling. System design leads engineers from single-analysis optimization to multidisciplinary optimization (MDO). An MDO is based on a compound model which can be seen as black boxes composition. A black box (called inner model in the following) can be a parameterized finite-element method (FEM) resolution as well as an analytical model.

Two pieces of information are needed to perform an optimal gradient-based sensitivity analysis of a compound model. First is composition information (what is the information exchanged between inner models). Second is the global sensitivities which will be studied (depending on optimizable inputs, constraints outputs and objective function). If both are known, a global sensitivity equation [1] can be used to perform an optimal-sensitivity analysis. Unfortunately, some optimization structures do not necessarily know both, as the one used in this paper.

When composing models based on different analysis software, we are facing with the implementation of the glue, which enables communication with other models and with the optimization software. This glue is a generally hand-made computer program because it depends on connected computing models, but it may be automated for specified communication protocol like file exchanges [5].

Manuscript received July 1, 2003.

B. Delinchant and F. Wurtz are with the Electrotechnical Laboratory of Grenoble (LEG), 38402 Saint Martin d'Héres cedex, France (e-mail: benoit.delinchant@leg.ensieg.inpg.fr; frederic.wurtz@leg.ensieg.inpg.fr).

E. Atienza is with the Design Processing Technologies Society, 38240

Meylan, France (e-mail: eric.atienza@ designprocessing.com).

Digital Object Identifier 10.1109/TMAG.2004.824907
Sensitivity evaluations being time consuming, several methods may improve the global analysis. Coding sensitivity at the level of inner models allows specific sensitivity analysis, like adjoin field technique for magnetic field problems solving [2]. However, in the case of compound model, an efficient analysis is not sufficient if it is called unnecessarily. Improving global time cost of compound model sensitivity analysis at the level of the glue has then to be considered.

The paper highlights some generic implications of model composition for sensitivity analysis. It proposes several techniques, trying to reduce the analysis number for each inner model. Some results are then presented, corresponding to the sizing (with an optimization software: Pro@Design) ${ }^{1}$ of a model composed of an FEM resolution (Flux2 $\mathrm{D}^{2}$ software) and analytical equations.

\section{Sensitivity Propagation Inside A CoMPound Model}

As studied in [3], two kinds of sensitivity propagation mechanisms can be considered to build global sensitivities. A forward mode crosses the dependency graph from inputs to outputs. This graph is made of nodes, which are inner models, and arcs, which are connections between nodes. A backward mode crosses the graph from outputs to inputs.

\section{A. Sensitivity Propagation Using Partial Derivatives}

Two sensitivities information may be considered crossing the graph. First can be partial derivatives (e.g., $\partial O_{1} / \partial I_{1}$ ), which require the knowledge of graph branches

$$
\frac{\partial O_{1}}{\partial I_{1}}=\sum_{b r_{1,1}}\left[\prod_{n_{k} \in b r_{1,1}} \frac{\partial O_{k}}{\partial I_{k}}\right]
$$

where

$b r_{1,1}$ branches from global Input 1 to Ouput 1 ( $I_{1}$ to $O_{1}$ ); $n_{k} \quad$ nodes of each branches $b r_{1,1}$;

$I_{k}, O_{k}$ input and output branches value at node $k$.

Indeed, the global-differentiation algorithm must know branches from input to output in forward mode, or from output to input in backward mode.

In the following example, the partial derivative is the composition of each inner partial derivative (see Fig. 1):

$$
\frac{d O_{1}}{d I_{1}}=\frac{\partial O_{1}}{\partial P_{1}}+\frac{\partial O_{1}}{\partial P_{3}} \cdot \frac{d P_{3}}{d P_{2}}
$$

where $P_{i}$ is the intermediary parameter number $i$.

\footnotetext{
${ }^{1}$ http://www.designprocessing.com/.

${ }^{2}$ http://www.cedrat.com/.
} 


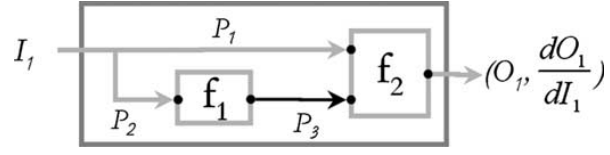

Fig. 1. Compound model based on partial derivative propagation.

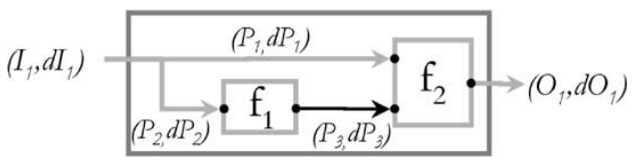

Fig. 2. Compound model based on differential propagation.

\section{B. Sensitivity Propagation Using Differentials}

Differentials can be considered as a possible second propagation information. A total differential is defined by

$$
d O_{j}=\sum_{i} \frac{\partial O_{j}}{\partial I_{i}} \cdot d I_{i}
$$

Propagation of differential in forward mode does not require any information about a dependency graph. Indeed, as shown in Fig. 2, differentials can be propagated with parameter values leading to the automatic evaluation of output differentials

$$
\begin{aligned}
& \left\{\begin{array}{l}
P_{3}=f_{1}\left(P_{2}\right) \\
d P_{3}=d f_{1}\left(P_{2}, d P_{2}\right)
\end{array}\right. \\
& \left\{\begin{array}{l}
O_{1}=f_{2}\left(P_{1}, P_{3}\right) \\
d O_{1}=d f_{2}\left[P_{1}, P_{3}, d P_{1}, d P_{3}\right] .
\end{array}\right.
\end{aligned}
$$

\section{Choosing a Sensitivity Propagation Method}

Each method owns its advantages and disadvantages. For instance, using graph knowledge may improve computation time when only some specific output sensitivities are analyzed.

The choice of forward differential propagation (Fig. 2) has been made (in the optimization software Pro@Design) to build a network which "only depends on local information" [4]. This means that adding a model in the beginning of the propagation process does not affect following connections.

Taking this property into account, we will now study how to implement sensitivity analysis at the level of inner model to optimize time of the global sensitivity evaluation.

To deal with this issue, three methods are proposed in the following, using a finite-difference method to illustrate our purpose. Some techniques, like the adjoin field technique, can be considered with the same approach, but is not treated in this paper.

\section{Three Sensitivity Analysis Methods}

\section{A. Method Using Differentials}

To perform the sensitivity analysis during an optimization iteration, inner models may be evaluated several times. Indeed, the size of the Jacobian matrix depends on the optimization specifications. The key to an optimized analysis is then to remember some information between each evaluation along iteration.

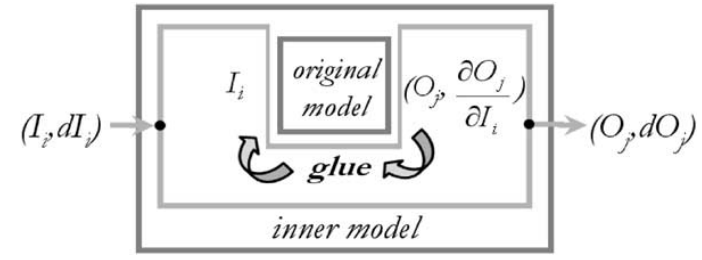

Fig. 3. Adapting differential propagation method to partial derivative sensitivity analysis of inner models thanks to glue code.

A first significant improvement is to remember the computation point (list of input and corresponding output values). Indeed, using sensitivity method such as forward finite difference requires only one extra model evaluation per derivative.

The following expression of the output differential is based on a directional derivative finite difference, and requires computing inner model and only one extra model evaluation

$$
d S=\left(S\left(I_{1}+\frac{d I_{1}}{p}, \ldots, I_{n}+\frac{d I_{n}}{p}\right)-S\left(I_{1}, \ldots, I_{n}\right)\right) \cdot p .
$$

Parameter " $p$ " is a normalization parameter which can be as following: $p=\sqrt{d I_{1}^{2}+\cdots+d I_{n}^{2}}$ or $p=\sum\left|d I_{i} / I_{i}\right|$.

This configuration of differentials computation and propagation gives a quick sensitivity analysis due to the independency of model call number in relation to optimizable input number. Despite its advantage, this method has a bad robustness because directional derivative mixes all input differentials together in the finite difference step [see (5)].

\section{B. Method Using Partial Derivatives}

Unfortunately, lots of models compute a sensitivity analysis based on partial derivatives. Glue must then be defined to create the required inner model with differentials (see Fig. 3).

Differential calls must be transformed into partial derivative calls and results must be returned into differential forms. The differential is then made of the sum of systematic partial derivations, which are built with the following finite-difference method:

$$
\begin{aligned}
d S_{j} & =\sum_{i} \frac{\partial S_{j}}{\partial E_{i}} \cdot d E_{i} \\
\frac{\partial S_{j}}{\partial I_{i}} & =\frac{S_{j}\left(I_{1}, \ldots, I_{i}+\Delta_{i}, \ldots, I_{n+p}\right)-S_{j}\left(I_{1}, \ldots, I_{i}, \ldots, I_{n+p}\right)}{\Delta_{i}} .
\end{aligned}
$$

This method increases the model call number due to the dependency of this number to the optimizable inputs $\left(d I_{i} \neq 0\right)$, (7).

\section{Method Reminding Computed Partial Derivatives}

During optimization iteration, the same local partial derivative may be used to compute different global partial derivatives, depending on the linkage of variables before the corresponding inner model.

In the following example (Fig. 4), the optimizer is looking for the gradient of $O_{1}$ depending on $I_{1}$ and $I_{2}$. Two calls are performed on the global model to compute these two partial derivatives. First call (9) allows building $f_{2}$ Jacobian and part 


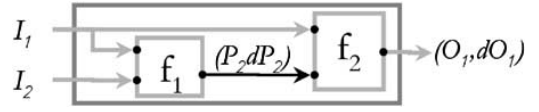

Fig. 4. Example in which a local partial derivative is used twice during the global Jacobian evaluation.

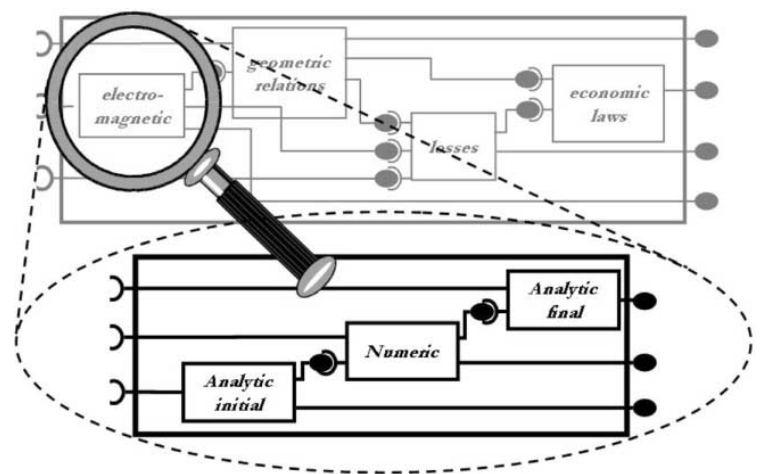

Fig. 5. Composition of models to create a global model of a transformer which can be used to perform optimizations.

of the $f_{1}$ one. Second call (10) can use part of the $f_{2}$ Jacobian to perform only one analysis on $f_{1}$

$$
\begin{aligned}
& \frac{\partial O_{1}}{\partial I_{1}}=\frac{\partial O_{1}}{\partial I_{1}}+\frac{\partial O_{1}}{\partial P_{2}} \frac{d P_{2}}{d I_{1}} \\
& \frac{\partial O_{1}}{\partial I_{2}}=\frac{\partial O_{1}}{\partial P_{2}} \frac{\partial P_{2}}{\partial I_{2}} .
\end{aligned}
$$

An improvement can be done by memorizing local Jacobians along the global sensitivity analysis. Indeed, during global sensitivity analysis, the model performs the analysis of several local partial derivatives, which can be stored in memory and reused for other global sensitivity analysis.

Thus, partial derivatives of the inner model are only computed once, reducing the model evaluation number by a factor which depends on the coupling between inner models.

These three methods will then be compared for the design of a transformer using such a compound model.

\section{APPLiCATION ON A TRANSFormer Design}

\section{A. Transformer Model Description and Building}

A compound model (FEM + analytic) of a transformer has been produced. To do so, a visual composer has been used, which enables to create a global model from the connection of several models [7].

Fig. 5 describes the composition of four models, (electromagnetic, geometric, losses, and economic) where the electromagnetic part is composed of a numeric model, based on an FEM software [5].

Analytical expressions are well suited to models like economical, losses, or geometrical ones. However, an FEM model is considered to take into account some phenomenon like coil border effects (Fig. 6) in the leakage reactance analysis.

Each analytical inner model is able to compute sensitivity thanks to symbolic differentiation and is based on differentials evaluation. Unfortunately, the numerical model is not dedicated to compute sensitivities and is considered as a black box, whose content can not be modified.

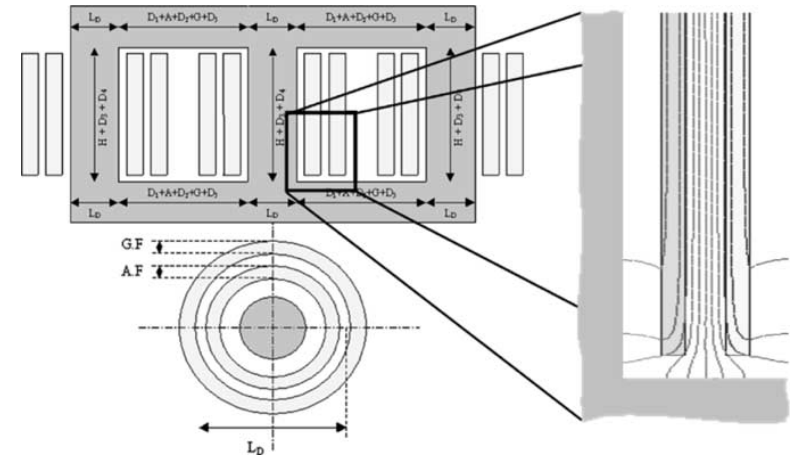

Fig. 6. Using the FEM model to ensure precision of leakage reactance evaluation.

TABLE I

Model Parameters Constraining the Sizing Process

\begin{tabular}{ll|ll} 
Inputs & Outputs \\
\hline $\mathrm{H}:$ & Coil height $(\mathrm{m})$ & $\mathrm{X}_{1}:$ & Primary leakage reactance $(\%)$ \\
$\mathrm{J}:$ & Current density $\left(\mathrm{A} / \mathrm{m}^{2}\right)$ & $\mathrm{A}:$ & Leg width $(\mathrm{m})$ \\
$\mathrm{N}_{1}:$ & Coil turn number & $\mathrm{Ltt}:$ & Magnetic circuit total length $(\mathrm{m})$ \\
$\mathrm{Bt}:$ & Flux density $(\mathrm{T})$ & cost $:$ & Transformer cost for 30 years of \\
& & & use (French francs).
\end{tabular}

TABLE II

TRANSFOMER OPTIMIZATION OF 5 SPECIFICATION SHEETS WITH 3 SENSITIVITY ANALYSIS METHOD. COMPUTER USED WAS A P-IV 1-GHz RAM 512 Mo

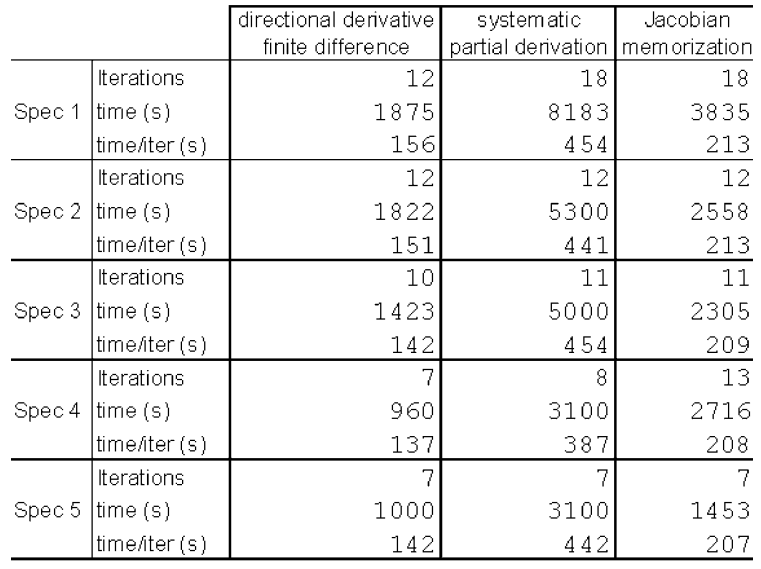

The aim of this study is, therefore, to test the three previous methods to implement sensitivities over the numerical model.

\section{B. Transformer Sizing Specifications and Performances}

Five specification sheets have been applied on the global model to optimize the transformer [6]. Constrained inputs and outputs, as well as the objective function (cost), are shown in Table I.

The three methods proposed in this paper were applied to perform a sensitivity analysis of the FEM model and performances are resumed in Table II.

The first method uses directional derivative finite difference. Parameter " $p$ " of (5) was defined twice to ensure stable results for the sensitivity computation. Indeed, regarding to various input model values, this method is hard to configure. However, when the appropriate normalization parameter " $p$ " is found, this 


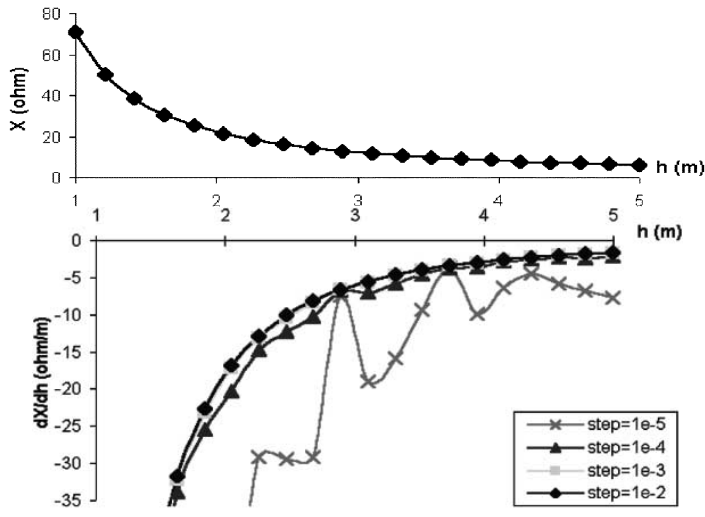

Fig. 7. (a) Leakage reactance depending on the transformer height. (b) Finite-difference step influence on sensitivity analysis precision of leakage reactance depending on the transformer height.

method give the quickest sensitivity analysis over the five specifications (Table II, column 1).

The second method uses systematic partial derivative. This technique is more robust because sensitivities are configured and computed separately. However, regarding the previous method, this one is three to four times slower (Table II, column 2).

The third method, which uses Jacobian memorization, has the stability advantage of the previous one and is two times quicker in this study (Table II, column 3).

\section{Sensitivity Analysis Configuration}

We saw that each sensitivity analysis method needs to be configured. The first method, using directional derivative finite difference, is configured by the normalization parameter " $p$ " used in (5). For our transformer model sensitivity analysis and with specific constrained inputs which are defined in Table II, normalization $p=\sqrt{d I_{1}^{2}+\cdots+d I_{n}^{2}}$ does not work. Then, normalization $p=\sum\left|d I_{i} / I_{i}\right|$ was tried to take into account huge differences between each differentials. This last formula gave stable results for the sensitivity analysis.

For the two last methods, the step of the finite difference has to be chosen regarding to numerical remeshing errors. This pa- rameter is easier to change than normalization formula of the first method. Indeed, some analysis are sufficient to find an appropriate step $\left(10^{-3}\right.$ for instance) (Fig. 7).

\section{CONCLUSION}

This paper has highlighted issues and given solutions to reduce the time cost taken by compound model sensitivity analysis, during an optimization process. This time cost has been reduced in a particular study by a factor of two with local Jacobian reminding, then by three with the differentials method.

Naturally, the total optimization time depends on the sensitivity quality, so these methods can not be applied without checking this one. Moreover, sensitivity analysis is subject to numerical errors, especially with the finite differences we used in this study. Robustness and configuration easiness are then important factors to choose one particular method.

Our study can be extended to other sensitivity methods, like adjoin field techniques, which can be used and optimized as well as finite difference, ensuring specific sensitivity analysis at the level of inner models.

\section{REFERENCES}

[1] J. Sobieszczanski-Sobieski, "Sensitivity of complex, internally coupled systems," AIAA J., vol. 28, no. 1, pp. 153-160, Jan. 1990.

[2] D. Ioan, I. Munteanu, and G. Ciuprina, "Magnetics adjoin field technique applied in optimal design of a nonlinear inductor," IEEE Trans. Magn., vol. 34, pp. 2849-2852, Sept. 1998.

[3] Gilbert, J. Charles-Le Vey, and J. Georges-Masse, "La differentiation automatique de fonctions représentées par des programmes" (in French), in Proc. INRIA RR-1557, Nov. 1991 , p. 68.

[4] E. Atienza, J. Bigeon, F. Wurtz, and B. Belhabib, "Steps to an electrical design environment," in Proc. IEEE-IECON, San Jose, CA, Nov. 29-Dec. 3, 1999.

[5] B. Delinchant, F. Wurtz, and J. Bigeon, "Automating tool integration to preliminary design request," in Proc. 10th Biennial IEEE Conf. Electromagnetic Field Computation, Perugia, Italy, June 16-19, 2002.

[6] B. Delinchant, F. Wurtz, and J. Fandino, "Mixing of FEM and analytical modeling for the preliminary design of a transformer," in Proc. 7th Int. Workshop Optimization and Inverse Problems in Electromagnetism, Lodz, Poland, Sept. 12-14, 2002.

[7] B. Delinchant, F. Wurtz, and D. Magot, "Simulation component composition for electrical systems design," Simulation: Trans. Soc. Modeling and Simulation Int., Special Issue: Component-Based Modeling and Simulation, submitted for publication. 